\title{
Improving LDA Topic Models for Microblogs via Tweet Pooling and Automatic Labeling
}

\author{
Rishabh Mehrotra \\ BITS Pilani \\ Pilani, India \\ erishabh@gmail.com \\ Wray Buntine \\ NICTA \& ANU \\ Canberra, Australia \\ Wray.Buntine@nicta.com.au lexing.xie@anu.edu.au
}

\begin{abstract}
Twitter, or the world of 140 characters poses serious challenges to the efficacy of topic models on short, messy text. While topic models such as Latent Dirichlet Allocation (LDA) have a long history of successful application to news articles and academic abstracts, they are often less coherent when applied to microblog content like Twitter. In this paper, we investigate methods to improve topics learned from Twitter content without modifying the basic machinery of LDA; we achieve this through various pooling schemes that aggregate tweets in a data preprocessing step for LDA. We empirically establish that a novel method of tweet pooling by hashtags leads to a vast improvement in a variety of measures for topic coherence across three diverse Twitter datasets in comparison to an unmodified LDA baseline and a variety of pooling schemes. An additional contribution of automatic hashtag labeling further improves on the hashtag pooling results for a subset of metrics. Overall, these two novel schemes lead to significantly improved LDA topic models on Twitter content.
\end{abstract}

\section{Categories and Subject Descriptors}

I.2.7 [Artificial Intelligence]: Natural Language Processing-Text analysis; H.3.3 [Information Storage And Retrieval]: Information Search and Retrieval-Clustering

\section{Keywords}

Topic modeling, LDA, Microblogs

\section{INTRODUCTION}

The "undirected informational" search task, where people seek to better understand the information available in document corpora, often uses techniques such as multidocument summarisation and topic modeling. Topic models uncover the salient patterns of a collection under the mixed-membership assumption: each document can exhibit multiple patterns to different extents. When analysing

Permission to make digital or hard copies of all or part of this work for personal or classroom use is granted without fee provided that copies are not made or distributed for profit or commercial advantage and that copies bear this notice and the full citation on the first page. Copyrights for components of this work owned by others than ACM must be honored. Abstracting with credit is permitted. To copy otherwise, or republish, to post on servers or to redistribute to lists, requires prior specific permission and/or a fee. Request permissions from permissions@acm.org.

SIGIR'13, July 28-August 1, 2013, Dublin, Ireland.

Copyright 2013 ACM 978-1-4503-2034-4/13/07 ...\$15.00.
Table 1: Example Topic Words

\begin{tabular}{|c|c|}
\hline Unpooled LDA (poor) & Proposed approach (coherent) \\
\hline $\begin{array}{c}\text { barack cool apple health iphone } \\
\text { los barackobama video uma gop }\end{array}$ & $\begin{array}{c}\text { flu swine news pandemic health } \\
\text { death flight h1n1 vaccine confirmed }\end{array}$ \\
\hline
\end{tabular}

text, these patterns are represented as distributions over words, called topics. Probabilistic topic models such as Latent Dirichlet Allocation (LDA) [1] are a class of Bayesian latent variable models that have been adapted to model a diverse range of document genres.

We consider the application of LDA to Twitter content, which poses unique challenges different to much of standard NLP content. Posts are (1) short - 140 characters or less, (2) mixed with contextual clues such as URLs, tags, and Twitter names, and (3) use informal language with misspelling, acronyms and nonstandard abbreviations (e.g. O o haha wow). Hence, effectively modeling content on Twitter requires techniques that can readily adapt to this unwieldy data while requiring little supervision.

Unfortunately, it has been found that topic modeling techniques like LDA do not work well with the messy form of Twitter content [15]. Topics learned from LDA are formally a multinomial distribution over words, and by convention the top-10 words are used to identify the subject area or give an interpretation of a topic. The naïve application of LDA to Twitter content produces mostly incoherent topics. Table 1 shows examples of poor topic words by directly applying LDA and topic words which are much more coherent and interpretable.

How can we extract better topics in microblogging environments with standard LDA? While linguistic "cleaning" of text could help somewhat, for instance [3], a complementary approach using LDA is also needed because there are so few words in a tweet. An intuitive solution to this problem is tweet pooling $[13,5]$ : merging related tweets together and presenting them as a single document to the LDA model. In this paper we examine existing tweet-pooling schemes to improve LDA topic quality and propose a few novel schemes. We compare the performance of these methods across three datasets, constructed to be representative of the diverse content collections in the microblog environment. We examine a variety of topic coherence evaluation metrics, including the ability of the learned LDA topics to reconstruct known clusters and the interpretability of these topics via statistical information measures.

Overall, we find that the novel method of pooling tweets by hashtags yields superior performance for all metrics on all datasets, and an automatic hashtag assignment scheme further improves the hashtag pooling results on a subset of metrics. Hence this work 
provides two novel methods for significantly improving LDA topic modeling on microblog content.

\section{TWEET POOLING FOR TOPIC MODELS}

The goal of this paper is to obtain better LDA topics from Twitter content without modifying the basic machinery of standard LDA. As noted in Section 1, microblog messages differ from conventional text: message quality varies greatly, from newswire-like utterances to babble. To address the challenges, we present various pooling schemes to aggregate tweets into "macro-documents" for use as training data to build better LDA models. The motivation behind tweet pooling is that individual tweets are very short $(\leq 140$ characters) and hence treating each tweet as an individual document does not present adequate term co-occurence data within documents. Aggregating tweets which are similar in some sense (semantically, temporally, etc.) enriches the content present in a single document from which the LDA can learn a better topic model. We next describe various tweet pooling schemes.

Basic scheme - Unpooled: The default treats each tweet as a single document and trains LDA on all tweets. This serves as our baseline for comparison to pooled schemes.

Author-wise Pooling: Pooling tweets according to author is a standard away of aggregating Twitter data $[13,5]$ and shown to be superior to unpooled Tweets. To use this method, we build a document for each author which combines all tweets they have posted.

Burst-score wise Pooling: A trend on Twitter [7] (sometimes referred to as a trending topic) consists of one or more terms and a time period, such that the volume of messages posted for the terms in the time period exceeds some expected level of activity. In order to identify trends in Twitter posts, unusual "bursts" of term frequency can be detected in the data. We run a simple burst detection algorithm to detect such trending terms and aggregate tweets containing those terms having high burst scores. To identify terms that appear more frequently than expected, we will assign a score to terms according to their deviation from an expected frequency. Assume that $M$ is the set of all messages in our tweets dataset, $R$ is a set of one or more terms (a potential trending topic) to which we wish to assign a score, and $d \in D$ represents one day in a set $D$ of days. We then define $M(R, d)$ as the subset of Twitter messages in $M$ such that (1) the message contains all the terms in $R$ and (2) the message was posted during day $d$. With this information, we can compare the volume in a specific day to the other days. Let $\operatorname{Mean}(R)=\frac{1}{|D|} \Sigma_{d \in D} M(R, d)$. Correspondingly, $S D(R)$ is the standard deviation of $M(R, d)$ over the days $d \in D$. The burstscore is then defined as:

$$
\text { burst-score }(R, d)=\frac{|M(R, d)-\operatorname{Mean}(R)|}{S D(R)}
$$

Let us denote an individual term having burst-score greater than some threshold $\tau$ on some day $d \in D$ as a burst-term. Then our first novel aggregation method of Burst Score-wise Pooling aggregates tweets for each burst-term into a single document for training LDA, where we found $\tau=5$ to provide best results. If any tweet has more than one burst-term, this tweet gets added to the tweet-pool of each of those burst-terms.

Temporal Pooling: When a major event occurs, a large number of users often start tweeting about the event within a short period of time. To capture such temporal coherence of tweets, the fourth scheme and our second novel pooling proposal is known as Temporal Pooling, where we pool all tweets posted within the same hour.

Hashtag-based Pooling: A Twitter hashtag is a string of characters preceded by the hash (\#) character. In many cases hashtags can be
Table 2: Datasets

\begin{tabular}{|l|l|}
\hline Dataset & Term/\% \\
\hline Generic & $\begin{array}{l}\text { music/17.9 business/15.8 movie/14.5 design/10.8 food/9.6 fun/9.1 health/6.9 family/6.4 } \\
\text { sport/4.9 space/3.2 }\end{array}$ \\
Specific & $\begin{array}{l}\text { Obama/23.2 Sarkozy/0.4 baseball/3.5 cricket/1.8 Mcdonalds/1.5 Burgerking/0.5 Ap- } \\
\text { ple/16.3 Microsoft/6.8 United-states/40.7 France/4.9 }\end{array}$ \\
Events & $\begin{array}{l}\text { Flight-447/0.9 Jackson/13.9 Lakers/13.8 attack/13.8 scandal/4.1 swine-flu/13.8 reces- } \\
\text { sion/12.3 conference/14.1 T20/4.4 Iran-election/8.6 }\end{array}$ \\
\hline
\end{tabular}

viewed as topical markers, an indication to the context of the tweet or as the core idea expressed in the tweet, therefore hashtags are adopted by other users that contribute similar content or express a related idea. One example of the use of hashtags is "ask GAGA anything using the tag \#GoogleGoesGaga for her interview! RT so every monster learns about it!! " referring to an exclusive interview for Google by Lady Gaga (singer). For the hashtag-based pooling scheme, we create pooled documents for each hashtag. If any tweet has more than one hashtag, this tweet gets added to the tweet-pool of each of those hashtags.

Other Pooling: While a few other combinations of pooling schemes (eg.author-time, hashtag-time, etc) are possible, the initial results obtained were not as good as those presented for the currently outlined pooling schemes.

\section{TWITTER DATASET CONSTRUCTION}

We construct three diverse datasets from a $20-30 \%$ sample of public Twitter posts in 2009. ${ }^{1}$ We chose one or two term queries (often with similar pairs of queries to encourage a non-strongly diagonal confusion matrix) to search a tweet collection and each resulting set of tweets was labeled by the query that retrieved it. Since the number of queries (equivalently the number of clusters) is known beforehand, we could use this knowledge to evaluate how well the topics output by LDA match with known clusters. A brief description of the three datasets is as follows:

Generic Dataset: 359,478 tweets from 11 Jan'09 to 30 Jan'09. A general dataset with tweets containing generic terms.

Specific Dataset: 214,580 tweets from 11 Jan'09 to 30 Jan'09. A dataset composed of tweets that refer to named entities.

Event Dataset: 207,128 tweets from 1 Jun'09 to 30 Jun'09. A dataset composed of tweets pertaining to specific events. The query terms represent these events and the time period was chosen specifically due to the high number of co-occurring events being discussed in this month relative to other months.

Table 2 provides the exact query terms and the percentage of tweets in the datasets retrieved by each query. Typically, less than one percent of tweets were retrieved by more than one query with the highest case of $4.6 \%$ overlap occurring in the generic dataset for the two queries "family" and "fun". We have removed tweets retrieved by more than one query in order to preserve uniqueness of tweet labels for later analysis with clustering metrics.

\section{EVALUATION METRICS}

Because there is no single method for evaluating topic models, we evaluate a range of metrics including those used in clustering (purity and NMI) and semantic topic coherence and interpretability (PMI) as discussed below.

In order to cluster with LDA, we let a topic represent each cluster and assign each tweet to its corresponding mixture topic of highest probability (an inferred quantity via LDA). Then by analysing clustering-based metrics, we wish to understand how well the different tweet pooling schemes are able to reproduce clusters representing the original queries used to produce the datasets.

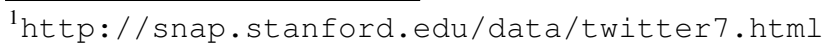


Formally, let $T_{i}$ be the set of tweets in LDA topic cluster $i$ and $Q_{j}$ be the set of tweets with query label $j$. Then let $T=\left\{T_{1}, \ldots, T_{|T|}\right\}$ be the set of all $|T|$ clusters and $Q=\left\{Q_{1}, \ldots, Q_{|Q|}\right\}$ be the set of all $|Q|$ query labels. Now we define our clustering-based metrics as follows.

Purity: To compute purity [6], each LDA topic cluster is assigned the query label most frequent in the cluster. Purity then simply measures the average "purity" of each cluster, i.e., the fraction of tweets in a cluster having the assigned cluster query label. Formally:

$$
\operatorname{Purity}(T, Q)=\frac{1}{|T|} \Sigma_{i \in\{1 \ldots|T|\}} \max _{j \in\{1 \ldots|Q|\}}\left|T_{i} \cap Q_{j}\right|
$$

Obviously, high purity scores reflect better original cluster reconstruction.

Normalized Mutual Information (NMI): As a more informationtheoretic measure of cluster quality, we also evaluate normalized mutual information (NMI) defined as follows

$$
N M I(T, Q)=\frac{2 I(T ; Q)}{H(T)+H(Q)},
$$

where $I(\cdot, \cdot)$ is mutual information, $H(\cdot)$ is entropy as defined in [6], $T$ and $Q$ are as defined previously. NMI is always a number between 0 and 1 and will be 1 if the clustering results exactly match the category labels while 0 if the two sets are independent.

As an alternative to clustering quality, we can instead measure topic interpretability or coherence, which is a human-judged quality that depends on the semantics of the words, and cannot be measured by model-based statistical measures that treat the words as exchangeable tokens. It is possible to automatically measure topic coherence with near-human accuracy [10] using a score based on pointwise mutual information (PMI). We use this to measure coherence of the topics from different tweet-pooling schemes.

Pointwise Mutual Information (PMI): PMI is a measure of the statistical independence of observing two words in close proximity where the PMI of a given pair of words $(u, v)$ is $\operatorname{PMI}(u, v)=$ $\log \frac{p(u, v)}{p(u) p(v)}$. Both probabilities are determined from the empirical statistics of the full collection, and we treat two words as cooccurring if both words occur in the same tweet.

For a topic $T_{k}\left(k \in\left\{1 \ldots\left|T_{k}\right|\right\}\right)$, we measure topic coherence as the average of PMI for the pairs of its ten highest probability words $\left\{w_{1}, \ldots, w_{10}\right\}$ :

$$
\operatorname{PMI-Score}\left(T_{k}\right)=\frac{1}{100} \Sigma_{i=1}^{10} \Sigma_{j=1}^{10} P M I\left(w_{i}, w_{j}\right) .
$$

The average of the PMI score over all the topics is used as the final measure of the PMI score.

\section{RESULTS FOR POOLING SCHEMES}

In this section we discuss the results of the experimental evaluation of the tweet pooling schemes introduced in Section 2. The datasets used were described in Section 3 while the evaluation metrics used were described in Section 4.

\subsection{Document Characteristics}

We first look at characteristics of the documents in the different pooling schemes for the three datasets as they may affect LDA. Table 3 presents these statistics.

The statistics presented above highlight the differences in the characteristics of the documents on which LDA models have been trained. The number of documents is the largest for the Unpooled and Author-wise schemes and smallest for the Hourly scheme, while the corresponding size of the documents displays an inverse relationship. On average the document size increases by a factor of seven in Hashtag-based pooling when compared against Unpooled or Author-wise pooling schemes. On the other extreme lies the Hourly pooling scheme with many fewer documents of a much larger average size.

\subsection{Comparison of Pooling Schemes}

For the three datasets (viz. Generic, Specific and Events) and four pooling schemes (plus Unpooled), we evaluate Purity scores, NMI scores and PMI scores obtained by training LDA for 10 topics using each setup. As seen in Table 4, the Hashtag-based pooling scheme clearly performs better than the Unpooled scheme as well as other pooling schemes.

\section{AUTOMATIC HASHTAG LABELING}

Hashtag-based pooling is clearly the best pooling scheme based on the results of Table 4, yet if we examine how many tweets have hashtags, we find the following distribution: generic $-22.3 \%$, specific, specific $-9.4 \%$, event $-19.5 \%$. In short, the majority of our data is not being used effectively by Hashtag-based pooling. Consequently, we conjecture that automatically assigning hashtags to some tweets should improve the overall evaluation metrics. Next we present an algorithm for performing this automated hashtag assignment with a tunable confidence threshold.

Hashtag Labeling Algorithm: First we pool all tweets by existing hashtags. Now, if the similarity score between an unlabeled and labeled tweet exceeds a certain confidence threshold $C$, we assign the hashtag of the labeled tweet to the unlabeled tweet (and hence it joins the pool for this hashtag). For our similarity metric between tweets, two obvious candidates are cosine similarity using TF and TF-IDF vector space representations [12].

On an initial exploratory analysis, we found that a medium-range confidence threshold of $C=0.5$ to give best results for both Purity and NMI. This is because tweets with the same class label have a higher average TF-IDF similarity than tweets with a different class label, so pooling these tweets together makes more topic-aligned hashtag pools that aid cluster reconstruction. On the other hand, PMI improved most for a rather high confidence threshold of $C=$ 0.9 since otherwise, tweets that were only marginally relevant to a hashtag reduced the overall topical coherence of hashtag-pooled documents and led to a noisier LDA model.

The overall results obtained via hashtag assignment are shown in Table 5 and demonstrate that Hashtag-based pooling with TFsimilarity tag assignment results in the best cluster reproductions with the highest Purity and NMI scores of all methods examined, while simple Hashtag-based pooling without tag assignment generally provides best results for topic coherence measured via PMI.

\section{RELATED WORK}

Our work is different from many pioneering studies on topic modeling of Tweets, as we focus on how to improve clustering metrics and topic coherence with existing algorithms. Prior work on topic modeling for tweets includes the following: [11] provide a scalable implementation of a partially supervised learning model (Labeled LDA). [5] provide a topical classification of Twitter users and messages. [4] propose a novel method for normalising ill-formed out-of-vocabulary words in short microblog messages. The TwitterRank system [13] and [5] used Author-wise pooling to apply LDA to tweets. [15] compared topic characteristics between Twitter and a traditional news medium - the New York Times; they propose to use one topic per tweet (similar to PLSA), and argue that this is better than the Unpooled LDA scheme and the author-topic model. [9] proposed two methods to regularize the resulting topics towards better coherence. [8] used retweet as an indicator of "interesting- 
Table 3: Document Characteristics for different pooling schemes.

\begin{tabular}{|l|ccc|ccc|ccc|}
\hline Pooling Scheme & \multicolumn{3}{|c|}{ \#of docs } & \multicolumn{3}{c|}{ Avg \# of words/doc } & \multicolumn{3}{c|}{ Max \# of words/doc } \\
\hline & Generic & Specific & Events & Generic & Specific & Events & Generic & Specific & Events \\
\hline Authorwise & 208300 & 118133 & 67387 & 17.6 & 20.4 & 15.4 & 4893 & 3586 & 2775 \\
\hline Unpooled & 359478 & 214580 & 207128 & 10.2 & 10.9 & 9.7 & 35 & 49 & 32 \\
\hline Burst Score & 7658 & 7436 & 5434 & 76.5 & 154.2 & 71.6 & 61918 & 420249 & 57794 \\
\hline Hourly & 465 & 464 & 463 & 8493.4 & 5387.5 & 2422 & 20144 & 18869 & 38893 \\
\hline Hashtag & 8535 & 7029 & 4099 & 70.4 & 187.2 & 78.4 & 61918 & 420249 & 57794 \\
\hline
\end{tabular}

Table 4: Results of different pooling schemes.

\begin{tabular}{|l|ccc|cccc|c|c|}
\hline Scheme & \multicolumn{3}{|c|}{ Purity } & \multicolumn{3}{c|}{ NMI Score } & \multicolumn{2}{c|}{ PMI score } \\
\hline & Generic & Specific & Events & Generic & Specific & Events & Generic & Specific & Events \\
\hline Unpooled & $0.49 \pm 0.08$ & $0.64 \pm 0.07$ & $0.69 \pm 0.09$ & $\mathbf{0 . 2 8} \pm \mathbf{0 . 0 4}$ & $0.22 \pm 0.05$ & $0.39 \pm 0.07$ & $-1.27 \pm 0.11$ & $0.47 \pm 0.12$ & $0.47 \pm 0.13$ \\
\hline Author & $\mathbf{0 . 5 4} \pm \mathbf{0 . 0 4}$ & $0.62 \pm 0.05$ & $0.60 \pm 0.06$ & $0.24 \pm 0.04$ & $0.17 \pm 0.04$ & $0.41 \pm 0.06$ & $0.21 \pm 0.09$ & $0.79 \pm 0.15$ & $0.51 \pm 0.13$ \\
\hline Hourly & $0.45 \pm 0.05$ & $0.61 \pm 0.06$ & $0.61 \pm 0.07$ & $0.07 \pm 0.04$ & $0.09 \pm 0.04$ & $0.32 \pm 0.05$ & $-1.31 \pm 0.12$ & $0.87 \pm 0.16$ & $0.22 \pm 0.14$ \\
\hline Burstwise & $0.42 \pm 0.07$ & $0.60 \pm 0.04$ & $0.64 \pm 0.06$ & $0.18 \pm 0.05$ & $0.16 \pm 0.04$ & $0.33 \pm 0.04$ & $0.48 \pm 0.16$ & $0.74 \pm 0.14$ & $0.58 \pm 0.16$ \\
\hline Hashtag & $\mathbf{0 . 5 4} \pm \mathbf{0 . 0 4}$ & $\mathbf{0 . 6 8} \pm \mathbf{0 . 0 3}$ & $\mathbf{0 . 7 1} \pm \mathbf{0 . 0 4}$ & $\mathbf{0 . 2 8} \pm \mathbf{0 . 0 4}$ & $\mathbf{0 . 2 3} \pm \mathbf{0 . 0 3}$ & $\mathbf{0 . 4 2} \pm \mathbf{0 . 0 5}$ & $\mathbf{0 . 7 8} \pm \mathbf{0 . 1 5}$ & $\mathbf{1 . 4 3} \pm \mathbf{0 . 1 4}$ & $\mathbf{1 . 0 7} \pm \mathbf{0 . 1 7}$ \\
\hline
\end{tabular}

Table 5: Overall percentage improvement of Hashtag-based pooling variants over Unpooled scheme.

\begin{tabular}{|c|c|c|c|c|c|c|c|c|c|}
\hline Pooling Scheme & \multicolumn{3}{|c|}{ Purity } & \multicolumn{3}{|c|}{ NMI Scores } & \multicolumn{3}{|c|}{ PMI Scores } \\
\hline & Generic & Specific & Events & Generic & Specific & Events & Generic & Specific & Events \\
\hline Hashtag & $+8.16 \%$ & $+5.88 \%$ & $+2.89 \%$ & $-3.44 \%$ & $+4.54 \%$ & $+7.69 \%$ & $+161 \%$ & $+204 \%$ & $+127 \%$ \\
\hline Hashtag + Tag-Assignment (TF) & $+21 \%$ & $+12.5 \%$ & $+8.69 \%$ & $+20.6 \%$ & $+9.1 \%$ & $+12.8 \%$ & $+164 \%$ & $+155 \%$ & $+124 \%$ \\
\hline Hashtag + Tag-Assignment (TF-IDF) & $+12.2 \%$ & $+9.4 \%$ & $+4.34 \%$ & $+10.3 \%$ & $+4.5 \%$ & $+10.25 \%$ & $+155 \%$ & $+159 \%$ & $+100 \%$ \\
\hline
\end{tabular}

ness" to improve retrieval quality. This related research suggests a number of orthogonal methods that could be used to complement our Hashtag-based tweet pooling scheme.

For automatic hashtag labeling that proved crucial to improving topics in our Hashtag-based pooling model, additional features for hashtag assignment can be found in the comprehensive study [14], which can be leveraged in future extensions along with novel socialmedia based similarity metrics like those incorporating inverse author frequency [2] and other social network properties.

\section{CONCLUSION}

This paper presented a way of aggregating tweets in order to improve the quality of LDA-based topic modeling in microblogs as measured by the ability of topics to reconstruct clusters and topic coherence. The results presented in Table 4 on three diverse selections of Twitter data suggest the novel scheme of Hashtagbased pooling leads to drastically improved topic modeling over Unpooled and other schemes. The further addition of automatic TF similarity-based hashtag assignment to Hashtag-based pooling outperforms all other pooling strategies and the Unpooled scheme on cluster reconstruction metrics as shown in Table 5. In conclusion, these two novel schemes present LDA users with novel methods for significantly improving LDA topic modeling on Twitter without requiring any modification of the underlying LDA machinery.

\section{Acknowledgments}

NICTA is funded by the Australian Government as represented by the Department of Broadband, Communications and the Digital Economy and the Australian Research Council through the ICT Centre of Excellence program.

\section{REFERENCES}

[1] D. Blei, A. Ng, and M. Jordon. Latent Dirichlet allocation. volume 3, pages 993-1022, 2003.

[2] S. E. Chan, R. K. Pon, and A. F. Cárdenas. Visualization and clustering of author social networks. pages 30-31, Arizona, USA, 2006.
[3] B. Han, P. Cook, and T. Baldwin. Automatically constructing a normalisation dictionary for microblogs. In Proc. of EMNLP-CoNLL 2012, pages 421-432, Korea, 2012.

[4] B. Han, P. Cook, and T. Baldwin. Lexical normalisation of social media text. ACM Transactions on Intelligent Systems and Technology, 4(1), Feb. 2013.

[5] L. Hong and B. Davison. Empirical study of topic modeling in Twitter. 1st ACM Workshop on Social Media Analytics, 2010.

[6] C. Manning, P. Raghavan, and H. Schütze. Introduction to Information Retrieval. Cambridge University Press, 2008.

[7] M. Naaman, H. Becker, and L. Gravano. Hip and trendy: Characterizing emerging trends on Twitter. J. Am. Soc. Inf. Sci. Technol., 62(5):902-918, May 2011.

[8] N. Naveed, T. Gottron, J. Kunegis, and A. C. Alhadi. Searching microblogs: coping with sparsity and document quality. In CIKM '11, pages 183-188, 2011.

[9] D. Newman, E. Bonilla, and W. Buntine. Improving topic coherence with regularized topic models. NIPS, 2011.

[10] D. Newman, J. Lau, K. Grieser, and T. Baldwin. Automatic evaluation of topic coherence. NAACL, 2010.

[11] D. Ramage, S. Dumais, and D. Liebling. Characterizing microblogs with topic models. AAAI Conference on Weblogs and Social Media, 2010.

[12] G. Salton and M. McGill. Introduction to modern information retrieval. McGraw-Hill, 1983.

[13] J. Weng, E.-P. Lim, J. Jiang, and Q. He. Twitterrank: finding topic-sensitive influential twitterers. In WSDM'10, pages 261-270, 2010.

[14] L. Yang, T. Sun, M. Zhang, and Q. Mei. We know what @ you \#tag: does the dual role affect hashtag adoption? WWW'12, pages 261-270, 2012.

[15] W. X. Zhao, J. Jiang, J. Weng, J. He, E.-P. Lim, H. Yan, and $X$. Li. Comparing twitter and traditional media using topic models. In ECIR'11, pages 338-349, 2011. 